\title{
Reconstruction of Smooth and Discontinuous Components of Solutions to Linear Ill-Posed Problems
}

\author{
Corresponding Member of the RAS V. V. Vasin ${ }^{a, b}$ \\ Received August 8, 2012
}

DOI: $10.1134 / \mathrm{S} 1064562413010146$

\section{INTRODUCTION}

In applications, there arise ill-posed problems formulated in the form of a linear operator equation

$$
A u=f \text {, }
$$

whose solution contains discontinuities, kinks, close peaks, and other singularities against the background of a smooth part. In the case of an approximately given right-hand side $f_{\delta},\left\|f-f_{\delta}\right\| \leq \delta$, and an unbounded inverse operator $A^{-1}$, this situation leads to considerable difficulties in the construction of regularizing algorithms based on variational methods, specifically, on Tikhonov regularization. This is explained by the fact that a stabilizer with a strong regularizing effect "smoothes" the fine structure of the solution and a stabilizing functional intended for a discontinuous solution may result in a poorly approximated smooth component of the solution.

Studies related to the reconstruction of noisy images and signal processing (see, e.g., $[1,2])$ make wide use of a technique (as a rule, without any theoretical justification) according to which a stabilizer is constructed in the form of two functionals, one intended for the smooth component, and the other, for the component with singularities:

$$
\begin{gathered}
\min \left\{\left\|A\left(u_{1}+u_{2}\right)-f_{\delta}\right\|^{2}+\alpha\left[\Omega_{1}\left(u_{1}\right)+\Omega_{2}\left(u_{2}\right)\right]:\right. \\
\left.u_{1} \in U_{1}, u_{2} \in U_{2}\right\} .
\end{gathered}
$$

Here, the functional $\Omega_{1}$ is frequently specified as a Hilbert norm, for example, the $L_{2}$ norm, while $\Omega_{2}$ is defined as the total or classical variation or their smooth approximations.

In this work, in the multidimensional case, we prove the convergence of the Tikhonov method in the form of (2) with stabilizers $\Omega_{1}\left(u_{1}\right)=\left\|u_{1}\right\|_{L_{q}}^{2}$ and

\footnotetext{
${ }^{a}$ Institute of Mathematics and Mechanics, Ural Branch, Russian Academy of Sciences, ul. S. Kovalevskoi 16, Yekaterinburg, 620219 Russia

${ }^{b}$ Ural Federal University, ul. Lenina 51, Yekaterinburg, 620083 Russia e-mail:vasin@imm.uran.ru
}

$\Omega_{2}\left(u_{2}\right)=\left\|u_{2}\right\|_{L_{p}}+J(u)$, where $J(u)$ is the total variation defined by the formula [3]

$J(u)=\sup \left\{\int_{D} u \operatorname{div} v d x: v \in C_{0}^{1}\left(D, R^{m}\right), \mid v(x) \leq 1\right\}$.

For a smooth function $u \in C^{1}(D)$, it becomes

$$
J(u)=\int_{D}|\nabla u| d x .
$$

Thus, problem (2) takes the form

$$
\begin{gathered}
\min \left\{\left\|A\left(u_{1}+u_{2}\right)-f_{\delta}\right\|_{F}^{2}+\alpha\left[\left\|u_{1}\right\|_{L_{q}}^{2}+\left(\left\|u_{2}\right\|_{L_{p}}^{2}+J\left(u_{2}\right)\right)\right]:\right. \\
\left.u_{1} \in U_{1}, u_{2} \in U_{2}\right\},
\end{gathered}
$$

where $U_{1}=L_{q}(D), U_{2}=\left\{u: u \in L_{p}(D), J(u)<\infty\right\}, F$ is an arbitrary normed space, and $1<p<q<\infty$. Here, in contrast to the traditionally used version, where only the total variation or the BV norm [4] is used with respect to $u_{2}$, we additionally introduced the $L_{p}$ norm, which ensures the strict convexity of the objective functional. As a result, the extreme element is unique, the components of the regularized solutions converge strongly in $L_{q}$ and $L_{p}$, and the variations converge as well.

\section{CONVERGENCE OF APPROXIMATE SOLUTIONS}

2.1. Multidimensional case. Let $A$ be a bounded linear operator from $L_{p}(D)$ to a normed space $F$, where $D$ is a subset of $R^{m}$. Assume that Eq. (1) has a unique solution $\hat{u}$ representable as a sum of two components: $\hat{u}=\hat{u}_{1}+\hat{u}_{2}$, where $\hat{u}_{1} \in L_{q}$ and $\hat{u}_{2} \in U_{2}=\left\{u: u \in L_{p}\right.$, $J(u)<\infty\}$. Obviously, this representation is not unique, since, along with $\hat{u}_{1}, \hat{u}_{2}$, the pair $\hat{u}_{1}+c, \hat{u}_{2}-c$ is also a solution. Nevertheless, the following result holds.

Lemma 1. There exists a unique pair $\hat{u}_{1}, \hat{u}_{2}$ that forms the solution $\hat{u}=\hat{u}_{1}+\hat{u}_{2}$ and is a minimizer in the problem 


$$
\begin{gathered}
\min \left\{\left\|u_{1}\right\|_{L_{q}}^{2}+\left\|u_{2}\right\|_{L_{p}}^{2}+J\left(u_{2}\right):\right. \\
\left.u_{1} \in L_{q}, u_{2} \in U_{2}, A\left(u_{1}+u_{2}\right)=f\right\}
\end{gathered}
$$

with a strictly convex objective functional.

Theorem 1. Under the assumptions made, for any $\alpha>0$, problem (4) has a unique solution $\left(u_{1}^{\alpha}, u_{2}^{\alpha}\right)$ such that, if the regularization parameter satisfies the relations

$$
\alpha(\delta) \rightarrow 0, \quad \frac{\delta^{2}}{\alpha(\delta)} \rightarrow 0, \quad \delta \rightarrow 0
$$

then

$$
\begin{gathered}
\lim _{\delta \rightarrow 0}\left\|u_{1}^{\alpha(\delta)}-\hat{u}_{1}\right\|_{L_{q}}=0, \lim _{\delta \rightarrow 0}\left\|u_{2}^{\alpha(\delta)}-\hat{u}_{2}\right\|_{L_{p}}=0, \\
\lim _{\delta \rightarrow 0} J\left(u_{2}^{\alpha(\delta)}\right)=J\left(\hat{u}_{2}\right) .
\end{gathered}
$$

Proof. Solvability. Since both components of any minimizing sequence $\left(u_{1}^{n}, u_{2}^{n}\right)$ in minimization problem (4) are bounded in $L_{q}$ and $L_{p}$, respectively, we see that, for each of them, there are weakly converging subsequences $\left(u_{1}^{n_{k}}, u_{2}^{n_{k}}\right)$ with limit points $\bar{u}_{1}, \bar{u}_{2}$.

Taking into account the weak continuity of the operator $A$, the weak lower semicontinuity of the $L_{p}$ and $L_{q}$ norms, and the lower semicontinuity of the total variation with respect to weak convergence in $L_{p}$ [4], we pass to the limit as $n \rightarrow \infty$ in the objective functional of problem (4) and conclude that $\left(\bar{u}_{1}, \bar{u}_{2}\right)$ is a minimizer in problem (4). The uniqueness of the minimizing pair follows from the strict convexity of the stabilizing functional.

Convergence. Redenoting the regularized solution $\left(\bar{u}_{1}, \bar{u}_{2}\right)$ by $\left(u_{1}^{\alpha}, u_{2}^{\alpha}\right)$ and using the extremeness property of this pair, we obtain the estimate

$$
\begin{gathered}
\left\|u_{1}^{\alpha}\right\|_{L_{q}}^{2}+\left\|u_{2}^{\alpha}\right\|_{L_{p}}^{2}+J\left(u_{2}^{\alpha}\right) \\
\leq \frac{\delta^{2}}{\alpha(\delta)}+\left\|\hat{u}_{1}\right\|_{L_{q}}^{2}+\left\|\hat{u}_{2}\right\|_{L_{p}}^{2}+J\left(\hat{u}_{2}\right),
\end{gathered}
$$

where the pair $\left(\hat{u}_{1}, \hat{u}_{2}\right)$ solves problem (5) and $\alpha(\delta)$ satisfies the conditions of the theorem.

Inequality (8) implies that the family $\left(u_{1}^{\alpha(\delta)}, u_{2}^{\alpha(\delta)}\right)$ is bounded and, hence, there exist weakly converging subsequences

$$
\begin{aligned}
& u_{1}^{\alpha\left(\delta_{k}\right)} \rightarrow \tilde{u}_{1} \text { (weakly) in } L_{q}(D), \\
& u_{2}^{\alpha\left(\delta_{k}\right)} \rightarrow \tilde{u}_{2} \text { (weakly) in } L_{p}(D) .
\end{aligned}
$$

Following the standard line of reasoning in Tikhonov regularization (see, e.g., $[4,5]$ ), we see that the sum of the limit points, i.e., $\tilde{u}=\tilde{u}_{1}+\tilde{u}_{2}$, solves the original equation (1).
Applying relations (8)-(10), the properties of $\left(\hat{u}_{1}, \hat{u}_{2}\right)$, Lemma 1 , and the assumptions of the theorem, we obtain the chain of inequalities

$$
\begin{gathered}
\left\|\hat{u}_{1}\right\|_{L_{q}}^{2}+\left\|\hat{u}_{2}\right\|_{L_{p}}^{2}+J\left(\hat{u}_{2}\right) \leq\left\|\tilde{u}_{1}\right\|_{L_{q}}^{2}+\left\|\tilde{u}_{2}\right\|_{L_{p}}^{2}+J\left(\tilde{u}_{2}\right) \\
\leq \liminf _{k \rightarrow \infty}\left[\left\|u_{1}^{\alpha\left(\delta_{k}\right)}\right\|_{L_{q}}^{2}+\left\|u_{2}^{\alpha\left(\delta_{k}\right)}\right\|_{L_{p}}^{2}+J\left(u_{2}^{\alpha\left(\delta_{k}\right)}\right)\right] \\
\leq \limsup _{k \rightarrow \infty}\left[\frac{\delta}{\alpha(\delta)}+\left\|\hat{u}_{1}\right\|_{L_{q}}^{2}+\left\|\hat{u}_{2}\right\|_{L_{p}}^{2}+J\left(u_{2}\right)\right] \\
=\left\|\hat{u}_{1}\right\|_{L_{q}}^{2}+\left\|\hat{u}_{2}\right\|_{L_{p}}^{2}+J\left(\hat{u}_{2}\right) .
\end{gathered}
$$

Since the stabilizer is strictly convex, these inequalities mean that $\tilde{u}_{1}=\hat{u}_{1}$, and $\tilde{u}_{2}=\hat{u}_{2}$. Moreover, since each the three terms of the stabilizing functional is weakly lower semicontinuous, it follows from (11) that

$$
\begin{gathered}
\lim _{k \rightarrow \infty}\left\|u_{1}^{\alpha\left(\delta_{k}\right)}\right\|_{L_{q}}=\left\|\hat{u}_{1}\right\|=\left\|\tilde{u}_{1}\right\|, \\
\lim _{k \rightarrow \infty}\left\|u_{2}^{\alpha\left(\delta_{k}\right)}\right\|_{L_{p}}=\left\|\hat{u}_{2}\right\|=\left\|\tilde{u}_{2}\right\|, \\
\lim _{k \rightarrow \infty} J\left(u_{2}^{\alpha\left(\delta_{k}\right)}\right)=J\left(\hat{u}_{2}\right) .
\end{gathered}
$$

Combining these relations with (9) and (10) and taking into account the uniqueness of the limit point $\left(\hat{u}_{1}, \hat{u}_{2}\right)$, we obtain (6) and (7), which complete the proof of the theorem.

Remark 1. Since the $L_{q}$ and $L_{p}$ norms are responsible for the smooth and nonsmooth components of the solution, respectively, it is reasonable to choose $q$ that is substantially larger than $p$. Moreover, depending on the information on the solution, we can use, as a stabilizer $\Omega_{1}\left(u_{1}\right)$, a stronger norm than the $L_{q}$ one, for example, the $W_{q}^{n}(D)$ norm $(n \geq 1)$ or the norm in the Lipschitz space. This guarantees the convergence of $u_{1}^{\alpha(\delta)}$ in $W_{q}^{n}(D)$ or its uniform convergence in the later case.

Consider a variant of Tikhonov regularization frequently occurring in applications. Specifically, let, in contrast to the previous case, only the total variation be used as a stabilizer with respect to the second component, i.e., $\Omega_{2}(u)=J(u)$. Assume additionally that the boundary of the domain $D \in R^{m}$ satisfies the cone condition and a constant is not a solution of the homogeneous equation $A u=0$. As before, $A$ is assumed to be an operator from $L_{p}(D)$ to a normed space $F$, but the parameter $p$ satisfies the constraint $1 \leq p \leq \frac{m}{m-1}$.

Theorem 2. For $\alpha>0$, the regularized problem has $a$ solution $\left(u_{1}^{\alpha}, u_{2}^{\alpha}\right)$ and, if the parameter satisfies the rela- 
tions $\alpha(\delta) \rightarrow 0, \frac{\delta^{2}}{\alpha(\delta)} \rightarrow 0$ as $\delta \rightarrow 0$, then the following properties hold:

(i) $\left\{u_{1}^{\alpha(\delta)}\right\}$ is relatively weakly compact in $L_{q}$.

(ii) $\left\{u_{2}^{\alpha(\delta)}\right\}$ is relatively compact for $1 \leq p<\frac{m}{m-1}$ and relatively weakly compact for $p=\frac{m}{m-1}$ in $L_{p}$.

(iii) If $\bar{u}_{1}$, and $\bar{u}_{2}$ are respective limit points of the sequences $u_{1}^{\alpha\left(\delta_{n}\right)}$ and $u_{2}^{\alpha\left(\delta_{n}\right)}$, then $\bar{u}=\bar{u}_{1}+\bar{u}_{2}$ is a solution of Eq. (1).

The proof is widely different from that of Theorem 1. We now need to use additional facts, such as the BVcoercivity of the classical Tikhonov functional (Lemma 4.1 in [4]) and the compactness of the embedding operator $I: \mathrm{BV} \rightarrow L_{p}$ (see [3, 4]). Here, $\mathrm{BV}$ is the space with the norm $\|u\|=\|u\|_{L_{1}}+J(u)$.

Thus, the stabilizing functional $\Omega(u)=\left\|u_{1}\right\|_{L_{q}}^{2}+$ $J\left(u_{2}\right)$ (in applications, typically, $q=2$ ) produces a substantially weaker stabilizing effect than $\Omega(u)=\left\|u_{1}\right\|_{L_{q}}^{2}+$ $\left\|u_{2}\right\|_{L_{p}}^{2}+J\left(u_{2}\right)$ (see problem (4)) and, additionally, the parameter $p$ is constrained.

2.2. One-dimensional case. In the case of spaces of one-variable functions $u(x)$ given on an interval $[a, b]$, the generalized variation (4) is denoted by $J_{a}^{b}$. In [5] the problem was studied in the traditional manner with a solution sought in the form of a single component $\left(u_{1}=0\right)$ and the regularized approximations generated by the variational method of [4] were proved to converge piecewise uniformly on each interval $\left[a^{\prime}, b^{\prime}\right] \subset$ $[a, b]$ where the solution of Eq. (1) is continuous. When the solution is sought in the form of two components as described above, a similar assertion holds for the second component.

Theorem 3. Let the conditions of Theorem 1 hold. Then, for $\alpha>0$, the regularized problem

$$
\begin{gathered}
\min \left\{\left\|A\left(u_{1}+u_{2}\right)-f_{\delta}\right\|^{2}+\alpha\left[\left\|u_{1}\right\|_{L_{q}}^{2}+\left(\left\|u_{2}\right\|_{L_{p}}^{2}+J_{a}^{b}\left(u_{2}\right)\right)\right]:\right. \\
\left.u_{1} \in L_{q}[a, b], u_{2} \in L_{p}[a, b]\right\}
\end{gathered}
$$

has a unique solution $\left(u_{1}^{\alpha}, u_{2}^{\alpha}\right)$ for which the claim of Theorem 1 on the convergence of $\hat{u}_{1}$, and $\hat{u}_{2}$ in $L_{q}$ and $L_{p}$, respectively, holds. Moreover,

$$
\forall x \in[a, b] \quad u_{2}^{\alpha(\delta)} \rightarrow \hat{u}_{2}(x) \text { as } \delta \rightarrow 0 .
$$

Additionally, for any interval $\left[a^{\prime}, b^{\prime}\right] \subseteq[a, b]$ not containing discontinuity points, the convergence in (13) is uniform in $x$. Here, $\left(\hat{u}_{1}, \hat{u}_{2}\right)$ is a pair satisfying Lemma 1 .

Proof sketch. Since the conditions of Theorem 1 hold, problem (12) has a unique solution $\left(u_{1}^{\alpha}, u_{2}^{\alpha}\right)$. The convergence of $\left(u_{1}^{\alpha(\delta)}, u_{2}^{\alpha(\delta)}\right)$ is proved by applying the technique of [5] modified with allowance for the features of the two-component problem under study. The proof relies heavily on the results of $[3$, Theorems 1.9 and 1.7; 6, Lemma 3.1], which concern functions of bounded total and classical variation (in the one-dimensional case).

Remark 2. When the regularized problem (4) is solved numerically, one needs a discrete approximation of the infinite-dimensional problem by a sequence of finite-dimensional problems. The stability of the general discrete approximation scheme and some nonsmooth optimization algorithms as applied to problem (4) were analyzed in [6, 7].

Remark 3. Solutions with singularities of various types arise not only in noisy image and signal processing, but also in many other applications, for example, in inverse problems of ground-based infrared atmospheric sounding [8], where one can also use the above-described technique for reconstructing a regularized solution in the form of two components.

\section{ACKNOWLEDGMENTS}

This study was supported by the Government of the Russian Federation (contract no. 11.G34.31.0064) at the Ural Federal University and by the Russian Foundation for Basic Research (project no. 12-01-00106) at the Institute of Mathematics and Mechanics, Ural Branch, Russian Academy of Sciences.

\section{REFERENCES}

1. M. Bertalmio, L. Wese, G. Sapiro, and S. Osher, IEEE Trans. Image Process. 12, 882-889 (2003).

2. E. J. Candes, J. Romberg, and T. Tao, Commun. Pure Appl. Math. 59, 1207-1223 (2006).

3. E. Giusti, Minimal Surfaces and Functions of Bounded Variations (Birkhäuser, Basel, 1984).

4. R. Acar and C. R. Vogel, Inverse Probl. 10, 1217-1229 (1994).

5. V. V. Vasin and M. A. Korotkii, J. Inverse Ill-Posed Probl. 15, 853-865 (2007).

6. V. V. Vasin, Proc. Steklov Inst. Math. Suppl. 1, 225-239 (2002).

7. V. V. Vasin, Dokl. Math. 63, 5-8 (2001).

8. K. G. Gribanov, V. I. Zakharov, G. G. Skorik, et al., Opt. Atmos. Okeana 17 (2), 124-127 (2011). 